\title{
Based on MATLAB and Adams new pruning cutting edge shape and shear force simulation
}

\author{
Hang Yin ${ }^{1, a}$, Zhaoxin Meng ${ }^{1}, Y u$ Zhang ${ }^{2}$, Bo $\mathrm{Yu}^{3}$ \\ ${ }^{1}$ School of mechanical and electrical engineering, Northeast Forestry University, Harbin, Heilongjiang Province, China \\ ${ }^{2}$ Research institute of internal and external decoration, Great Wall motor co. LTD, Bao Ding, Hebei Province, China \\ ${ }^{3}$ Division of structure design, Research institute of Harbin FRP, Harbin, Heilongjiang Province, China
}

\begin{abstract}
Pruning is an indispensable tool for gardening. The pruning mechanism of pruning scissors and the shape of the cutting edge directly affect the efficiency of shear. Therefore, it is of great significance to design a new type of pruning scissors to improve the efficiency of garden work. It accords with the concept of green manufacturing energy-saving and environmental protection. The blade shape directly affects the shear efficiency. Therefore, the blade of the best Angle can improve the shear efficiency and save energy. According to the motion track of the moving blade and the shear mode. Using MATLAB software to simulate the shape of the blade of the best Angle. This work is instructive to the design of the blade. The force of the blade in the working process of shear force reaction scissors. Using Adams software, the dynamic simulation of tree branch was carried out. It is helpful to verify the shear performance of new pruning shears.
\end{abstract}

\section{Introduction}

With the acceleration of urban landscaping process. The work load of garden care and modelling is increasing gradually. The use of pruning tools also increased, pruning tasks become heavy, pruning tools also consume more energy. Carbon emissions are also relatively high. Not accord with the concept of energy conservation and emission reduction. Pointing to this problem, it is of great significance to study and design a new energysaving and efficient pruning tool.

Because of the complexity of branches and branches and the diversity of tree species. There are many tools available on the market for pruning. There are many different principles and ways of cutting. Due to the complexity of the working environment of pruning tools and the particularity of working objects. Therefore, in order to meet the requirement of pruning operation. It is necessary to solve the problem whose designed structure is simple and compact and another problem which cost reasonable pruning tool head. To solve the intelligent level and cost of garden pruning tool and environmental protection restrict each other issues. By designing the whole structure of the new branch pruning shears, the cutting operation is cut off fast, high efficiency and low power consumption, increase utilization rate, reduce production cost. This is very useful for promotion.

\section{The structure design of new pruning shears}

Cutting blade is an effective part of scissors. It is mainly designed for the curved blade of the moving blade. The curvature of the blade has an important influence on the shear performance of the scissors. In the working process, the fixed tool holder mainly plays the role of holding and supporting. Therefore, we only need to analyze the moving blade. The force of the blade in the working process is very complicated. Therefore, we need to analyze the cutting principle of the cutter first. The force of the cutting tool is shown in Figure. 1 and Figure. 2

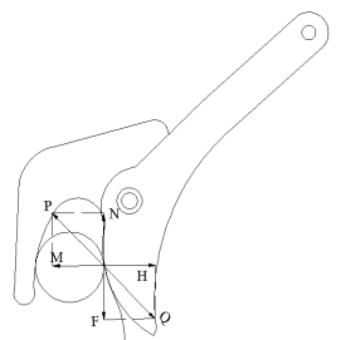

Figure. 1 Tool contact force

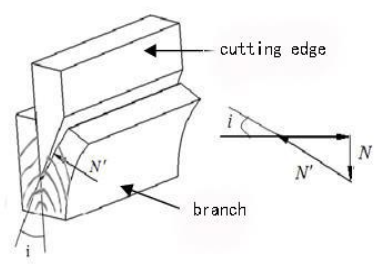

Figure. 2 Tool shear force
Shear force $P$ and roles of branches in the dynamic reaction force on the blade $Q$ for a pair of interaction force, shear force $P$ can be decomposed into the blade of the tangential force $N$ and the normal force $M$, reaction $Q$ can be decomposed into the friction of branches and blades $F$ and vertical reaction $H, I$ is the Angle between shear force and the vertical direction of cutting edge. scissors blade vertical Angle, $\varphi$ is friction Angle, $\varphi^{\prime}$ is friction Angle of the blade surface [1]. 


$$
\begin{gathered}
P=2 N^{\prime} \tan \varphi \\
2 N^{\prime}=\frac{N}{\sin \frac{i}{2}} \\
\tan \varphi^{\prime}=\frac{\tan \varphi}{\sin \frac{i}{2}} \\
P=N \tan \varphi^{\prime} \\
\varphi^{\prime}=\arctan \frac{\tan \varphi}{\sin \frac{i}{2}}
\end{gathered}
$$

If shearing motion, $P>Q_{\max }$ is required, the shear force is bigger than the maximum force of the blade. Therefore, the cutting condition is different:

(1) when $\alpha=0, \mathrm{M}=0$, is vertical cutting.

(2) when $0<\alpha \leq \varphi^{\prime}, 0<M \leq P_{\max }$, there is only a tendency rotary cutting, not to rotary cutting.

(3) when $\alpha>\varphi^{\prime}, M>P_{\max }^{\prime}$, rotary cutting.

Cutting methods are divided into three types: vertical cutting, cutting and rotary cutting. Course of practical application, rotary cutting is more labor-saving than cutting and vertical cutting. As a result, most of the tools of agro-forestry tools tend to rotary cutting. The blade of the new pruning shears also adopts the rotary cutting mode. So we should analyze the principle of vertical cutting. Firstly, the cutter is assumed as an idealized model, Ignore the micro-level of the blade. Simplify the moving blade into a wedge. The branches are assumed to be homogeneous material.

When begin rotary cutting, tangential force $M$ and normal force $N$ act on the branches, the blade moves along the resultant force of two forces, as is shown in the Figure.3, When the blade moves in a vertical direction for $H$, while it traveling $S_{0}$ in the horizontal direction. Under the force of friction $f, m^{\prime}$ stop at one side of frictional Angle $\varphi^{\prime}$.Branches move $\mathrm{S}$ in the horizontal direction relative to the moving blade. We define the Angle of rotary cutting as the Angle between the motion direction of the moving blade relative to the branch and the normal line of the blade.

$$
\tan \alpha=\frac{S}{H}
$$

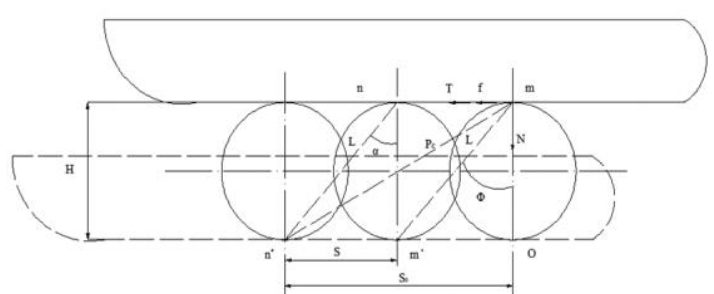

Figure. 3 Rotary cutting schematic diagram

According to the related literature of agroforestry machinery design [2], The range of the best rotary cutting Angle is between $35^{\circ}$ and $55^{\circ}$. Therefore, according to the actual situation and the analysis will be used as the Angle of rotary cutting. The motion diagram of the moving blade is shown in Figure. 4.

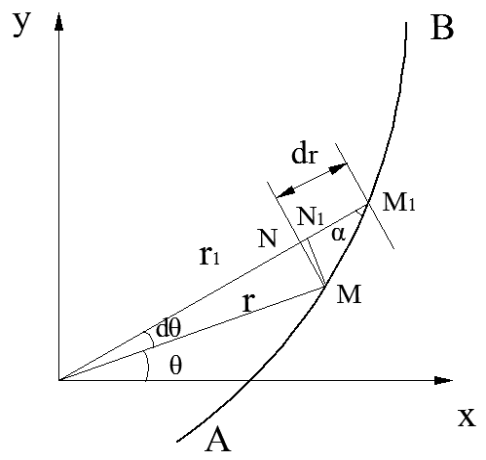

Figure. 4 Schematic diagram of moving blade shear branch

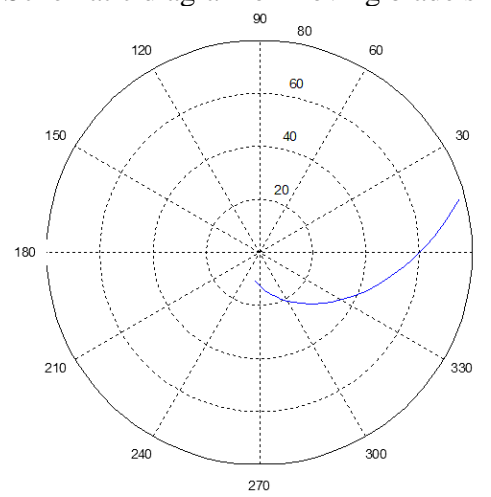

Figure. 5 The shape of the polar curve of the blade

The center of the rotating shaft of the moving blade coincides with the origin of the coordinate system, move the blade around the origin to a certain Angle. When the blade edge curve $\mathrm{AB}$ is rotated $\mathrm{d} \theta$ by $\theta$, point of tangency $\mathrm{M}$ turn into $M_{1}$, radius $\mathrm{r}$ turn into $r^{\prime}$, it increased. If $\mathrm{d} \theta$ goes to 0 , then $M N_{1}$ is equal to $M N$.

$$
\begin{gathered}
\tan \alpha=\frac{M N}{M N_{1}}=\frac{\mathrm{rd} \theta}{\mathrm{dr}} \\
\frac{1}{r} d r=\frac{1}{\tan \alpha} d \theta \\
\ln r=\theta+C
\end{gathered}
$$


Put Angle into the (8), take integrate both sides of this equation. In (9), $C$ is constant, when $\theta=0$, then $r_{0}=C$ [3].

According to (10), the trajectory of the spiral tangent curve is approximately a logarithmic curve. And it has the same geometrical characteristics as the Angle between the ray and the curve. The Angle is equal to the Angle of rotation. According to this conclusion, the shape of the blade curve can be designed.

The shape of the blade is designed according to the equation of rotary cutting Angle curve. The blade length is $60 \mathrm{~mm}$, rotary cutting Angle is $45^{\circ}$. The curve equation of the blade is obtained. The range of Angle $\theta$ is between $-110^{\circ}$ to $15^{\circ}$. Enter (10) into MATLAB, using MATLAB to draw the shape of the blade curve is shown in Figure .5[4].

$$
r=c e^{\frac{\theta}{\tan \alpha}}
$$

Because of the cutting diameter range of the new pruning shears is $0-30 \mathrm{~mm}$, the external cutting edge of the fixed tool holder should be greater than $30 \mathrm{~mm}$. The shape of some branches is irregular, so the external edge should leave allowance, design the external cutting edge of $35 \mathrm{~mm}$. Increase the stability of clamping branches, according to the maximum shear diameter. The optimum cutting position is suitable for the middle position of the hook slot. Therefore, the median diameter should be designed to be $32 \mathrm{~mm}$. The interior design of the hook groove should be relatively narrow. On the one hand, it is suitable for cutting the branch of the path, on the other hand, it plays a clamping effect on the large diameter branch. It's designed to be $25 \mathrm{~mm}$.

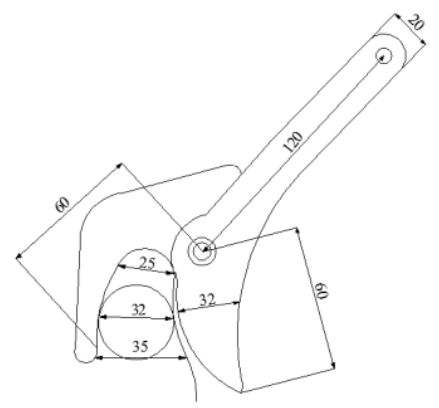

Figure. 6 New pruning cutting part structure and geometric dimension drawing.

The shape of the moving blade is shown in Figure.6. The design of arc blade can be used to rotary cut. This kind of cutting can reduce the shear force in a very short time. Improve the smoothness of shear and improve shear quality. The blade surface should cover the cross section of the whole cutting material. Because the main cutting position of the moving blade is located in the middle part of the pruning cutting, the width of the middle part should be greater than or equal to the branch section. The cutting range of pruning shears is $0-30 \mathrm{~mm}$. Shear should be cut completely, the design of the moving blade should be leave allowance, design its widest position is $32 \mathrm{~mm}$. The tip position of the moving blade should be swept over the hook tip position. Therefore, the distance between the rotation axis and the moving blade tip should be the same as that of the rotation axis to the hook tip. It is designed to be $60 \mathrm{~mm}$. The handle of moving blade is designed to be $120 \mathrm{~mm}$, the width is designed to be $20 \mathrm{~mm}$.

\section{The establishment of Adams virtual prototype and calculation of cutting force}

Adams software can simulate the dynamics of an organization, the detection of the impact force of the object can be well simulated. The maximum cutting force of the cutter can be obtained by measuring the cutting force of the cutter according to the impact force of the blade and the branch. Adams2014 software added flexible body simulation. Because the branches belong to the flexible body, it is more realistic to use Adams2014 software to simulate it.

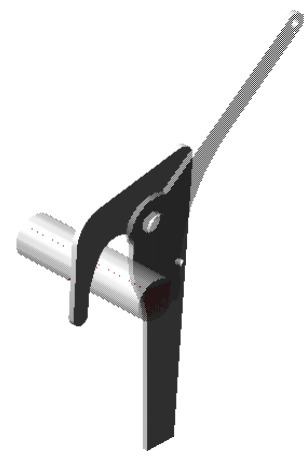

Figure. 7 Virtual prototype $3 \mathrm{~d}$ model establishment

Import model, constrain load and drive the model. Use Adams2014's new function Rigid to Flex Rigid body to transform the soft body function to transform the branch into a flexible body. The fixed tool holder and operation interface apply a fixed pair, a rotating pair is applied to the moving blade and the fixed tool holder, apply a drive on the rotate pair, speed setting is $30^{\circ} / \mathrm{s}$. Joint Motion and Modify Contact are shown in Figure. 8 and Figure. 9. A fixed pair is applied to the branch and the fixed tool holder. It is considered that the branches will not move, the branch and the blade apply a touch and choose Flex Body to Solid [5]. The constraint and load, as well as the driver loading, are shown in Figure. 10.

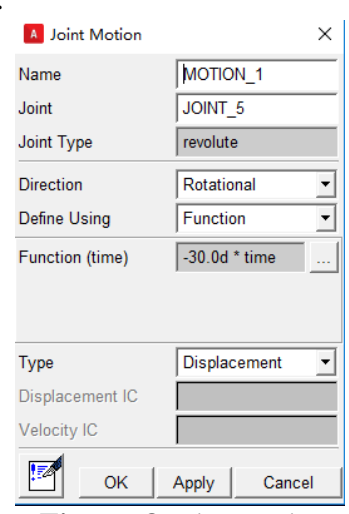

Figure. 8 Joint Motion

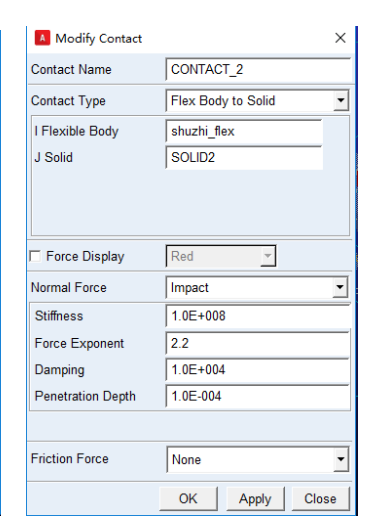

Figure. 9 Modify Contact 


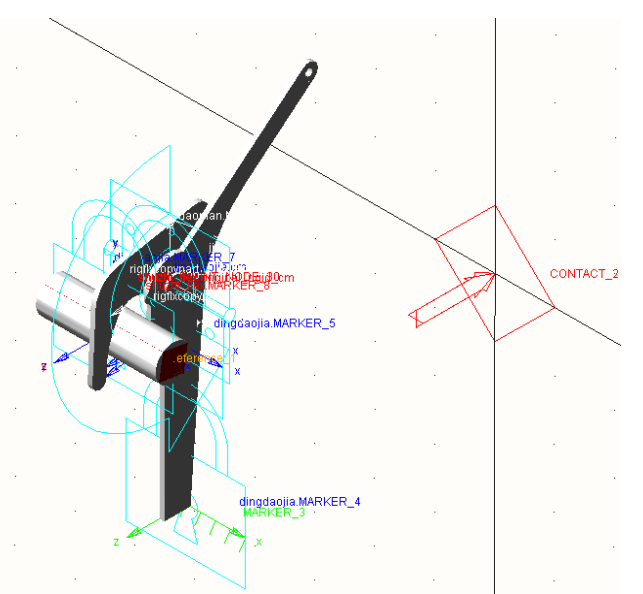

Figure. 10 Effect diagram of constraint load and drive loading.

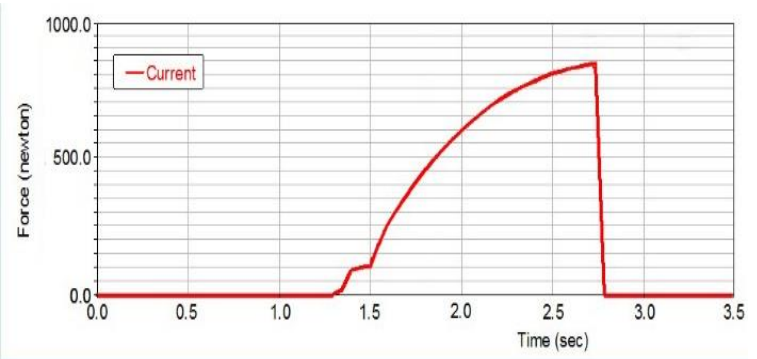

Figure. 11 Shear force curve

After solving the calculation, we found that it was set to $3 \mathrm{~s}$, and the blade was swept across the branch section in order to save the remaining amount. We set the time to $3.5 \mathrm{~s}$. Measure the contact force between the moving blade and the branch. The measurement results are shown in Figure. 11. Start at about 0-1.3s, the moving blade does not contact the branch, $1.3 \mathrm{~s}$ contact, there was a sudden change of force between 1.3-1.5s, the force gradually began to increase between $1.5 \mathrm{~s}$ and $2.7 \mathrm{~s}$. Tool contact force increased slowly to late stage, at the highest point, the maximum shear force is 848 N. Finally, the force decreased between $2.7 \mathrm{~s}$ and $2.8 \mathrm{~s}$, the branch was cut off in 2.8 seconds.

\section{Conclusion}

In this paper, the structural design of new pruning scissors is presented. The equation of the blade curve is derived, using MATLAB to draw the blade shape and Adams software to dynamic calculation. The maximum shear force at shear is $848 \mathrm{~N}$. It provides a basis for the verification of shear performance of new pruning shears.

\section{References}

1. Xiao Zhengfu, Liu Shuqin, Hu Yixuan. Branch cutting tools. Northeast Forestry University press, 1992

2. Chinese academy of agricultural mechanization sciences. Agricultural machinery design manual. China Agricultural Science and Technology press, 2007
3. Sun Liping, Cui Yongzhi, Liu Yixing. Analysis on the difference of radial chord to load in the compression process of branches. Forestry Science and Technology, 1996

4. Li lin. Study on the blade curve of the cutter blade of the disc cutter. Mechanization of Rural Pastoral Areas, 2010

5. Di Ping, Andrea Arenz. Modelling and control of a flexible "goliath" robot a case study using ADAMS/Control,13th European ADAMS Users Conference, 2008 